PROCEEDINGS OF THE

AMERICAN MATHEMATICAL SOCIETY

Volume 139, Number 8, August 2011, Pages 2681-2696

S 0002-9939(2011)10790-1

Article electronically published on February 8, 2011

\title{
WHAT IS A SYSTEM OF PARAMETERS?
}

\author{
LOUIZA FOULI AND CRAIG HUNEKE
}

(Communicated by Irena Peeva)

\begin{abstract}
In this paper we discuss various refinements and generalizations of a theorem of Sankar Dutta and Paul Roberts. Their theorem gives a criterion for $d$ elements in a $d$-dimensional Noetherian Cohen-Macaulay local ring to be a system of parameters, i.e., to have height $d$. We chiefly remove the assumption that the ring be Cohen-Macaulay and discuss similar theorems.
\end{abstract}

\section{INTRODUCTION}

One of the fundamental concepts in commutative algebra is that of height. Let $(R, \mathfrak{m})$ be a Noetherian local ring of dimension $d$. Every ideal $I$ of $R$ is generated by $d$ elements up to radical. The question of determining the height of ideals in general is then the same as that of determining the height of an ideal generated by $d$ elements. An interesting paper of Sankar Dutta and Paul Roberts 2 gives a criterion for $d$ elements in a Cohen-Macaulay local ring to be a system of parameters, i.e., to have maximal height. We recall their theorem: let $(R, \mathfrak{m})$ be a $d$-dimensional Cohen-Macaulay local ring, and let $x_{1}, \ldots, x_{d}$ be a system of parameters. Suppose that $\left(y_{1}, \ldots, y_{d}\right) \subset\left(x_{1}, \ldots, x_{d}\right)$. Write $y_{i}=\sum_{j} a_{i j} x_{j}$ (since this notation occurs throughout this paper, we abbreviate it by writing $(\underline{y}) \stackrel{A}{\subset}(\underline{x}))$, and let $\operatorname{det} A$ be the determinant of the matrix whose coefficients are the $\bar{a}_{i j}$. Since $\operatorname{det} A \cdot\left(x_{1}, \ldots, x_{d}\right) \subset$ $\left(y_{1}, \ldots, y_{d}\right)$, multiplication by $\operatorname{det} A$ induces a well-defined map from $R /\left(x_{1}, \ldots, x_{d}\right)$ to $R /\left(y_{1}, \ldots, y_{d}\right)$. The theorem of Dutta and Roberts states that this map is injective if and only if $y_{1}, \ldots, y_{d}$ form a system of parameters, i.e., if and only if the ideal $\left(y_{1}, \ldots, y_{d}\right)$ has height $d$. The fact that the map is injective if both $y_{1}, \ldots, y_{d}$ and $x_{1}, \ldots, x_{d}$ are regular sequences was well-known; it is the converse that was new.

In the 1970s, Hochster introduced the problem of understanding what constraints parameters in a Noetherian local ring must satisfy. This means understanding what equations cannot be satisfied by a system of parameters in a Noetherian local ring.

Received by the editors March 15, 2010 and, in revised form, August 2, 2010.

2010 Mathematics Subject Classification. Primary 13A35, 13C40, 13 D45.

Key words and phrases. System of parameters, local cohomology, limit closure, tight closure, plus closure.

The first author was partially supported by the NSF-AWM Mentoring Travel Grant, grant DMS-0839954. She thanks the Department of Mathematics at the University of Kansas for its hospitality.

The second author was partially supported by the National Science Foundation, grant DMS0756853.

(C) 2011 American Mathematical Society Reverts to public domain 28 years from publication 
The most famous such constraint is the monomial conjecture of Hochster. The theorem of Dutta and Roberts can be thought of as giving the opposite: it gives a relation which can only be satisfied by parameters. However, the Dutta-Roberts result leads to many interesting questions, some of which turn out to be closely related, in various guises, to the monomial conjecture. We will mention some of these questions in the last section. A first and obvious question is whether or not the same result holds without the Cohen-Macaulay assumption. We have been able to answer this for 1-dimensional rings (Theorem 4.1 and Example 4.3). Even this case is not trivial. We have no counterexample in higher dimensions.

Perhaps an even more basic question is whether or not the assumption of the theorem is independent of the matrix of coefficients chosen. For example, a famous special case of the theorem is the case in which $R$ is a polynomial ring, $k\left[x_{1}, \ldots, x_{d}\right]$ over a field of characteristic 0 , and $y_{1}, \ldots, y_{d}$ are homogeneous of degree $n_{1}, \ldots, n_{d}$, respectively. In this case Euler's formula gives that $y_{i}=\frac{1}{n_{i}} \sum_{1 \leq j \leq d} \frac{\partial y_{i}}{\partial x_{j}} x_{j}$, and by letting $\Delta$ be the determinant of this particular matrix of coefficients, one has that $\Delta \notin\left(y_{1}, \ldots, y_{d}\right)$ if and only if the height of $\left(y_{1}, \ldots, y_{d}\right)$ is $d[9]$. In the language above, the map from $R /\left(x_{1}, \ldots, x_{d}\right)$ to $R /\left(y_{1}, \ldots, y_{d}\right)$ is either injective or zero. Is this true for an arbitrary matrix of coefficients? Strooker [12] proved that independence is guaranteed if one changes the statement of the theorem to apply to the map induced by multiplication by the determinant from $R /\left(x_{1}, \ldots, x_{d}\right)^{\lim }$ to $R /\left(y_{1}, \ldots, y_{d}\right)^{\lim }$ (part of the statement is that this is a well-defined homomorphism). Here $R /\left(x_{1}, \ldots, x_{d}\right)^{\lim }$ (respectively $R /\left(y_{1}, \ldots, y_{d}\right)^{\lim }$ ) denotes the image of $R /\left(x_{1}, \ldots, x_{d}\right)$ (respectively $R /\left(y_{1}, \ldots, y_{d}\right)$ ) under the identification of $H_{\mathfrak{m}}^{d}(R)$ with the direct limit of $R /\left(x_{1}^{n}, \ldots, x_{d}^{n}\right)$ (respectively the image of $R /\left(y_{1}, \ldots, y_{d}\right)$ in $H_{\left(y_{1}, \ldots, y_{d}\right)}^{d}(R)$ under the identification of this module with the direct limit of $\left.R /\left(y_{1}^{n}, \ldots, y_{d}^{n}\right)\right)$. Thus the question of independence of the choice of matrix naturally leads to a consideration of other maps, and in particular brings into the picture local cohomology.

We begin this paper by proving that various maps are always injective if both the sequence $\underline{x}:=x_{1}, \ldots, x_{d}$ and the sequence $\underline{y}=y_{1}, \ldots, y_{d}$ form systems of parameters. Included in this list are the map above from $R /(\underline{x})^{\lim }$ to $R /(y)^{\lim }$ induced by multiplication by $\operatorname{det} A$ (this is now independent of the matrix of coefficients $\left(a_{i j}\right)$ chosen), a natural map from $H_{(\underline{x})}^{d}(R)$ to $H_{(\underline{y})}^{d}(R)$, and in positive prime characteristic, the map from $R /(\underline{x})^{*}$ to $R /(y)^{*}$ induced by multiplication by $\operatorname{det} A$, where the ${ }^{*}$ refers to tight closure. One can also replace the tight closure by plus closure (they are the same for parameters by [1], but are not necessarily the same for other ideals).

We next ask whether the obvious generalization of the theorem of Dutta and Roberts holds in the cases listed in the paragraph above: if the maps are injective, must the $y$ form a system of parameters? We are able to prove that there is a fixed power of $\mathfrak{m}$, independent of $\underline{x}$ and $y$, such that if all the $x_{i}$ are in this fixed power, then the natural generalization of the theorem of Dutta and Roberts holds in the first two maps of the paragraph above (Corollary 5.4). Moreover, if the ring is analytically irreducible, then we obtain a full generalization (Proposition 5.1). This is mainly due to the vanishing theorem of Hartshorne and Lichtenbaum, which can be thought of as giving a necessary and sufficient condition for $d$ elements $y$ in a complete local domain of dimension $d$ to be a system of parameters, namely, 
that $H_{(\underline{y})}^{d}(R) \neq 0$. We need $R$ to be equal characteristic or of dimension at most three in order to apply the monomial conjecture, which is known to hold in these cases [4. In the cases of tight closure and plus closure, we do not know whether injectivity on the maps forces the $y$ to be a system of parameters. We have been unable to prove this or give a counterexample.

As we mentioned above, a full generalization of the Dutta-Roberts theorem escapes us at the moment. Note, however, that the well-known direction of their theorem is false in the non-Cohen-Macaulay case, unlike the other maps discussed above. In fact we prove that for all systems of parameters $\underline{x}$ and every positive integer $t$ the induced map $R /(\underline{x}) \stackrel{\left(x_{1} \cdots x_{d}\right)^{t-1}}{\longrightarrow} R /(\underline{x})^{[t]}$ is injective if and only if the ring is Cohen-Macaulay (Proposition 6.3).

We do give the best possible answer for the limit closures. Our final result proves the following: let $(R, \mathfrak{m})$ be a local ring of equicharacteristic or of dimension at most three. Set $d=\operatorname{dim} R$. There exists an integer $\ell$ with the following property: whenever $\underline{x}=x_{1}, \ldots, x_{d}$ is a system of parameters with $(\underline{x}) \subset \mathfrak{m}^{\ell}$ and $\underline{y}=y_{1}, \ldots, y_{d}$ is a sequence of elements such that $(\underline{y}) \stackrel{A}{\subset}(\underline{x})$, then $\underline{y}$ is a system of parameters if and only if the map $R /(\underline{x})^{\lim \cdot \operatorname{det} A} \longrightarrow R /(y)^{\lim }$ is injective. We give an example to show that this result is not true without the parameters being "deep" enough inside the maximal ideal (Example 6.1).

\section{Preliminaries and Basic Results}

Let $\underline{x}=x_{1}, \ldots, x_{n}$ be a sequence of elements in a Noetherian ring $R$. Let $(\underline{x})$ denote the ideal generated by $x_{1}, \ldots, x_{n}$. We recall here that the limit closure of $(\underline{x})$ is given by

$$
(\underline{x})^{\lim }=\bigcup_{t \geq 1}\left(x_{1}^{t}, \ldots, x_{n}^{t}\right): x_{1}^{t-1} \cdots x_{n}^{t-1} .
$$

Before stating some of the conditions we will be studying, we need to recall some well-known definitions.

Definition 2.1. Let $R$ be a Noetherian local ring of positive characteristic $p$. An element $x$ is in the tight closure of an ideal $I$, denoted $I^{*}$, if there exists an element $c$, not in any minimal prime of $R$, such that for all large $q=p^{e}, c x^{q} \in I^{[q]}$, where $I^{[q]}$ is the ideal generated by all $f^{q}$ for $f \in I$.

Definition 2.2. Let $R$ be a Noetherian domain. The absolute integral closure of $R$, denoted $R^{+}$, is the integral closure of $R$ in a fixed algebraic closure of the fraction field of $R$.

Remark 2.3. Let $(R, \mathfrak{m})$ be a Noetherian local ring of dimension $d$. Let $\underline{x}=$ $x_{1}, \ldots, x_{d}$ be a system of parameters and let $\underline{y}=y_{1}, \ldots, y_{d}$ be a sequence of elements in $R$ such that $(\underline{y}) \subset(\underline{x})$. Let $A=\left(a_{i j}\right)$ be a matrix such that $y_{i}=\sum_{j=1}^{d} a_{i j} x_{j}$ and let $\operatorname{det} A$ denote the determinant of $A$. We will use the notation $(\underline{y}) \stackrel{A}{\subset}(\underline{x})$ to denote that $A$ is a matrix of coefficients relating $\underline{y}$ to $\underline{x}$. Consider the following statements, where in parts (3) and (4) we assume that $R$ has positive characteristic $p$, and in part (4) that $R$ is in addition a domain:

(1) The map $R /(\underline{x})^{\lim \cdot \operatorname{det} A} \longrightarrow R /(\underline{y})^{\lim }$ is injective. 
(2) The map $H_{(\underline{x})}^{d}(R) \longrightarrow H_{(\underline{y})}^{d}(R)$ is injective.

(3) The map $R /(\underline{x})^{*} \stackrel{\operatorname{det} A}{\longrightarrow} R /(\underline{y})^{*}$ is injective.

(4) The map $H_{(\underline{x})}^{d}\left(R^{+}\right) \longrightarrow H_{(\underline{y})}^{\bar{d}}\left(R^{+}\right)$is injective.

(5) The map $R /(\underline{x}) \stackrel{\cdot \operatorname{det} A}{\longrightarrow} R /(\underline{y})$ is injective.

(6) $\underline{y}$ is a system of parameters.

The maps on local cohomology are not yet defined, but will be defined in a way compatible with the maps on the lim closures. We wish to determine the relationship between these statements. In this section we will prove that the maps in parts (1) and (2) are injective if $y$ is a system of parameters. In a later section we will prove that the maps in (3) and (4) are injective if $y$ is a system of parameters. In contrast, the map in (5) does not necessarily have to be injective when (6) holds.

The fact that the map in (1) is well-defined (and does not depend on the specific matrix $A$ ) was proved by Strooker in 12 . We will prove this as well as a by-product of the following lemma, which we need to define the map in (2). Recall that if $M$ is a matrix, then $I_{1}(M)$ is the ideal generated by the entries of $M$.

Lemma 2.4. Let $R$ be a commutative ring with identity. Let $\left(G_{\bullet}, \Delta\right)$ and $\left(F_{\bullet}, \partial\right)$ be complexes of $R$-modules. Let $\alpha: G_{\bullet} \rightarrow F_{\bullet}$ be a map of complexes. Suppose that for all $0 \leq j \leq i-1$ there exist maps $\delta_{j}: G_{j} \rightarrow F_{j+1}$ such that $\alpha_{j}=\partial_{j+1} \delta_{j}+\delta_{j-1} \Delta_{j}$. We further assume that there exists a complex of free $R$-modules $\left(G^{\prime} \bullet, \Delta^{\prime}\right)$ and a map of complexes $\beta: G^{\prime} \bullet \rightarrow G_{\bullet}$ such that $I_{1}\left(\beta_{i}\right) H_{i}\left(F_{\bullet}\right)=0$. Then we may extend the homotopy to $\delta_{i}: G_{i}^{\prime} \rightarrow F_{i+1}$ for the map of complexes $\alpha \circ \beta: G^{\prime} \bullet \rightarrow F_{\bullet}$.

Proof. We have the following commutative diagram:

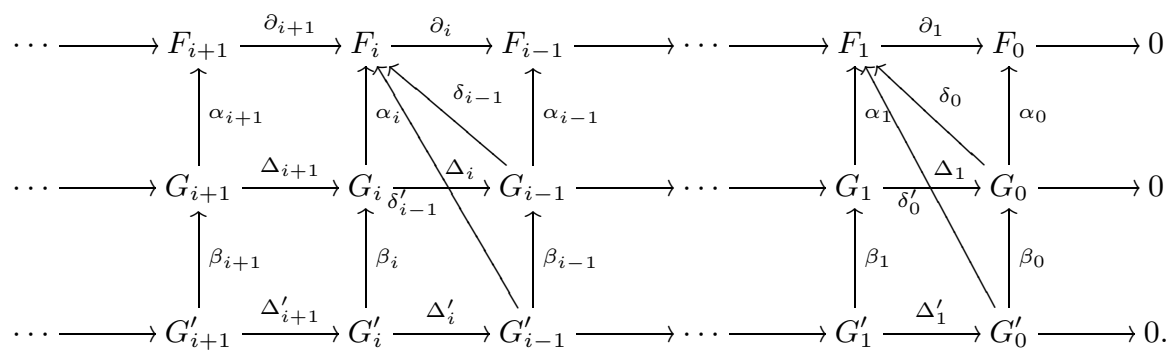

First we begin by showing the there is a homotopy for the map of complexes $\alpha \circ \beta: G^{\prime} \bullet \rightarrow F_{\bullet}$ up to level $i-1$. For $0 \leq j \leq i-1$ define $\delta_{j}^{\prime}=\delta_{j} \circ \beta_{j}$. Then $\delta_{j}^{\prime}: G^{\prime}{ }_{j} \rightarrow F_{j+1}$ and $\alpha_{j} \circ \beta_{j}=\left(\partial_{j+1} \delta_{j}+\delta_{j-1} \Delta_{j}\right) \beta_{j}=\partial_{j+1} \delta_{j} \beta_{j}+\delta_{j-1} \Delta_{j} \beta_{j}=$ $\partial_{j+1} \delta_{j}^{\prime}+\delta_{j-1} \beta_{j-1} \Delta_{j}^{\prime}=\partial_{j+1} \delta_{j}^{\prime}+\delta_{j-1}^{\prime} \Delta_{j}^{\prime}$.

We now claim that $\operatorname{Im}\left(\alpha_{i}-\delta_{i-1} \Delta_{i}\right) \subset \operatorname{ker} \partial_{i}$. Indeed,

$$
\begin{aligned}
\partial_{i}\left(\alpha_{i}-\delta_{i-1} \Delta_{i}\right) & =\partial_{i} \alpha_{i}-\partial_{i} \delta_{i-1} \Delta_{i}=\alpha_{i-1} \Delta_{i}-\partial_{i} \delta_{i-1} \Delta_{i} \\
& =\partial_{i} \delta_{i-1} \Delta_{i}+\delta_{i-2} \Delta_{i-1} \Delta_{i}-\partial_{i} \delta_{i-1} \Delta_{i}=0 .
\end{aligned}
$$

Finally we show that $\operatorname{Im}\left(\alpha_{i} \beta_{i}-\delta_{i-1}^{\prime} \Delta_{i}^{\prime}\right) \subset \operatorname{Im}\left(\partial_{i+1}\right)$. To see this notice that

$$
\begin{aligned}
\operatorname{Im}\left(\alpha_{i} \beta_{i}-\delta_{i-1}^{\prime} \Delta_{i}^{\prime}\right) & =\operatorname{Im}\left(\alpha_{i} \beta_{i}-\delta_{i-1} \beta_{i-1} \Delta_{i}^{\prime}\right)=\operatorname{Im}\left(\alpha_{i} \beta_{i}-\delta_{i-1} \Delta_{i} \beta_{i}\right) \\
& \subset \operatorname{Im}\left(\alpha_{i}-\delta_{i-1} \Delta_{i}\right) I_{1}\left(\beta_{i}\right) \subset \operatorname{Im}\left(\partial_{i+1}\right),
\end{aligned}
$$

since we have $I_{1}\left(\beta_{i}\right) H_{i}\left(F_{\bullet}\right)=0$. Therefore we may lift to a map $\delta_{i}^{\prime}: G_{i}^{\prime} \rightarrow F_{i+1}$ such that $\alpha_{i} \beta_{i}-\delta_{i-1}^{\prime} \Delta_{i}^{\prime}=\delta_{i}^{\prime} \partial_{i+1}$. 
As an immediate corollary, we get the following effective result which explains how the different choices of matrices relate to the multiplication maps of the determinants of the matrices.

Corollary 2.5. Let $R$ be a commutative ring with identity, $d=\operatorname{dim} R$ and let $\left(y_{1}, \ldots, y_{d}\right) \subset\left(x_{1}, \ldots, x_{d}\right)$. Suppose that there exist two matrices $A=\left(a_{i j}\right)$ and $B=\left(b_{i j}\right)$ such that $y_{i}=\sum_{j=1}^{d} a_{i j} x_{j}=\sum_{j=1}^{d} b_{i j} x_{j}$. Then $\left(y_{1} \cdots y_{d}\right)^{d-1}(\operatorname{det} A-\operatorname{det} B) \in$ $\left(y_{1}^{d}, \ldots, y_{d}^{d}\right)$.

Proof. Let $\underline{y}=y_{1}, \ldots, y_{d}$ and $\underline{x}=x_{1}, \ldots, x_{d}$. Consider the map of the Koszul complexes $\bar{K}_{\bullet}(\underline{y} ; R) \rightarrow K_{\bullet}(\underline{x} ; R)$ :

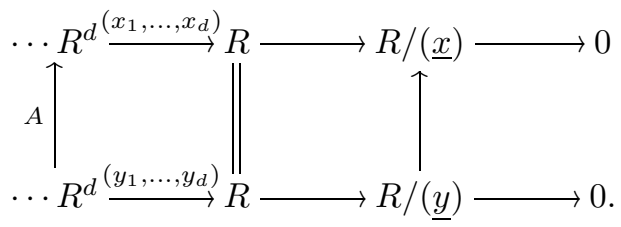

The map induced by $A$ extends to the whole Koszul complex by using exterior powers of $A, \bigwedge^{i} A: \bigwedge^{i}\left(R^{d}\right) \rightarrow \bigwedge^{i}\left(R^{d}\right)$. Similarly we obtain the map $K_{\bullet}(\underline{y} ; R) \stackrel{\wedge^{\bullet} B}{\rightarrow}$ $K_{\bullet}(\underline{x} ; R)$. Note that we have the following diagram:

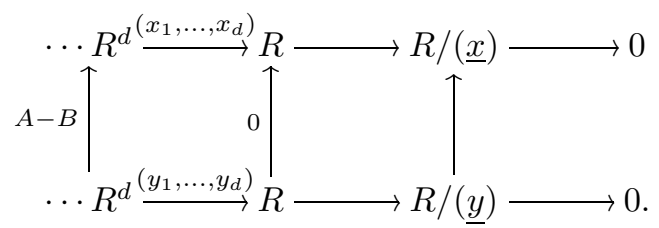

On the other hand $H_{\bullet}\left(K_{\bullet}(\underline{x} ; R)\right)$ is annihilated by $(\underline{x})$ and therefore it is also annihilated by $(\underline{y})$. We will begin defining a homotopy for the map of complexes $\bigwedge^{i}(A)-\bar{\Lambda}^{i}(B): K_{\bullet}(\underline{y} ; R) \rightarrow K_{\bullet}(\underline{x} ; R)$ by taking $\delta_{0}=0$. We compose it with $K_{\bullet}\left(y_{1}^{2}, \ldots, y_{d}^{2} ; R\right) \rightarrow K_{\bullet}(\underline{y} ; R)$ induced by $\wedge^{\bullet}\left(\begin{array}{ccc}y_{1} & & 0 \\ & \ddots & \\ 0 & & y_{d}\end{array}\right)$. Therefore by Lemma 2.4 we may extend the homotopy to $\delta_{1}$ for $K_{\bullet}\left(y_{1}^{2}, \ldots, y_{d}^{2} ; R\right) \rightarrow K_{\bullet}(\underline{x} ; R)$. Repeating the process we obtain

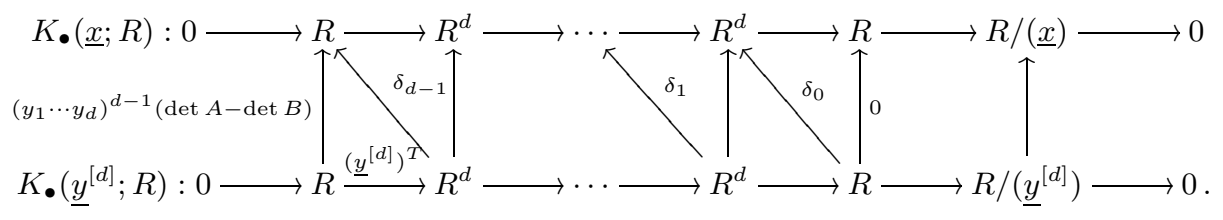

Hence $\left(y_{1} \cdots y_{d}\right)^{d-1}(\operatorname{det} A-\operatorname{det} B) \in\left(y_{1}^{d}, \ldots, y_{d}^{d}\right)$.

We can now give a quick proof for the map in part (1) of Remark 2.3 to be well defined. This is due to Strooker. See [12, 5.1.14-5.1.17].

Remark 2.6. Let $R$ be a commutative ring with identity, $d=\operatorname{dim} R$ and let $\underline{x}=$ $x_{1}, \ldots, x_{d}$ and $\underline{y}=y_{1}, \ldots, y_{d}$ be sequences of elements such that $(\underline{y}) \stackrel{A}{\subset}(\underline{x})$. Then 


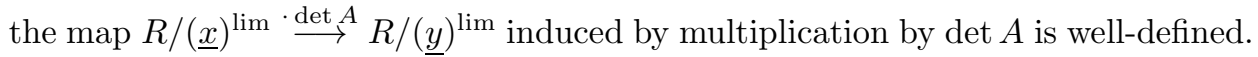
Moreover, this homomorphism does not depend on the choice of $A$. If $(\underline{y}) \stackrel{B}{\subset}(\underline{x})$, then multiplication by $\operatorname{det} B$ induces the same map.

Proof. Let $r \in(\underline{x})^{\lim }$. Then there exists a positive integer $t$ such that $r \cdot x_{1}^{t-1} \cdots x_{d}^{t-1}$ $\in\left(x_{1}^{t}, \ldots, x_{d}^{t}\right):=(\underline{x})^{[t]}$. Since $(\underline{y}) \subset(\underline{x})$, there exists a positive integer $s$ such that $(\underline{y})^{[s]} \subset(\underline{x})^{[t]}$. Let $D$ be a matrix such that $(\underline{y})^{[s]}=D(\underline{x})^{[t]}$. Let $C_{1}$ be the diagonal matrix with entries $y_{i}^{s-1}$ and let $C_{2}$ be the diagonal matrix with entries $x_{i}^{t-1}$. Notice that we have the following inclusions:

$$
(\underline{y})^{[s]} \stackrel{C_{1}}{\subset}(\underline{y}) \stackrel{A}{\subset}(\underline{x}) \text { and }(\underline{y})^{[s]} \stackrel{D}{\subset}(\underline{x})^{[t]} \stackrel{C_{2}}{\subset}(\underline{x}) .
$$

Let $C=A C_{1}$ and $E=C_{2} D$. Then by Corollary 2.5

$$
\left(y_{1} \cdots y_{d}\right)^{s d-s}(\operatorname{det} C-\operatorname{det} E) \in(\underline{y})^{[s d]} \text {. }
$$

Hence

$$
\operatorname{det} A\left(y_{1} \cdots y_{d}\right)^{s-1}\left(y_{1} \cdots y_{d}\right)^{s d-s}-\operatorname{det} D\left(y_{1} \cdots y_{d}\right)^{s d-s}\left(x_{1} \cdots x_{d}\right)^{t-1} \in(\underline{y})^{[s d]}
$$

and thus $r \operatorname{det} A\left(y_{1} \cdots y_{d}\right)^{s-1}\left(y_{1} \cdots y_{d}\right)^{s d-s}-r \operatorname{det} D\left(y_{1} \cdots y_{d}\right)^{s d-s}\left(x_{1} \cdots x_{d}\right)^{t-1} \in$ $(\underline{y})^{[s d]}$. Notice, however, that

$$
r \operatorname{det} D\left(y_{1} \cdots y_{d}\right)^{s d-s}\left(x_{1} \cdots x_{d}\right)^{t-1} \in \operatorname{det} D\left(y_{1} \cdots y_{d}\right)^{s d-s}(\underline{x})^{[t]},
$$

since $r \in(\underline{x})^{\lim }$. Also,

$$
\operatorname{det} D\left(y_{1} \cdots y_{d}\right)^{s d-s}(\underline{x})^{[t]} \subset\left(y_{1} \cdots y_{d}\right)^{s d-s}(\underline{y})^{[s]} \subset(\underline{y})^{[s d]} .
$$

Thus $r \operatorname{det} A\left(y_{1} \cdots y_{d}\right)^{s-1}\left(y_{1} \cdots y_{d}\right)^{s d-s}=r \operatorname{det} A\left(y_{1} \cdots y_{d}\right)^{s d-1} \in(\underline{y})^{[s d]}$. Therefore, $r \operatorname{det} A \in(\underline{y})^{\lim }$, and the map is then well defined.

The fact that the map does not depend on the choice of $A$ follows directly from Corollary 2.5. This says that the difference between the two determinants lies in $(\underline{y})^{\lim }$.

Next we define the maps from part (2) and equivalently part (4) from Remark 2.3 ,

Discussion 2.7. Let $R$ be a Noetherian local ring, $d=\operatorname{dim} R$ and let $\underline{x}=x_{1}, \ldots, x_{d}$ and $\underline{y}=y_{1}, \ldots, y_{d}$ be sequences of elements such that $(\underline{y}) \stackrel{A}{\subset}(\underline{x})$. We will define a homomorphism $\Phi: H_{(\underline{x})}^{d}(R) \longrightarrow H_{(\underline{y})}^{d}(R)$ such that the following diagram commutes:

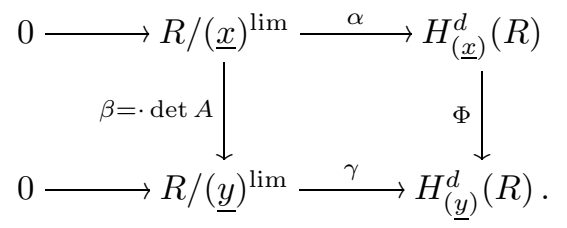

In other words, when we restrict $\Phi$ to the natural image of $R /(\underline{x})$ in $H_{(\underline{x})}^{d}(R)$, the map goes to $\operatorname{det} A$ times the image of $R /(\underline{y})$ in $H_{(\underline{y})}^{d}(R)$, i.e., is compatible with the map given in part (1) of Remark 2.3. To define $\Phi$, let $u \in H_{(\underline{x})}^{d}(R)$. Then $u=$ $\left[\frac{r}{x_{1}^{n} \cdots x_{d}^{n}}\right]$ for some $n$ and some $r \in R$. Since $(\underline{y}) \subset(\underline{x})$ there exist a matrix $B$ and 
a positive integer $s$ such that $(\underline{y})^{[s]}=B(\underline{x})^{[n]}$. We define $\Phi: H_{(\underline{x})}^{d}(R) \longrightarrow H_{(\underline{y})}^{d}(R)$ by $\Phi(u)=\left[\frac{r \operatorname{det} B}{y_{1}^{s} \cdots y_{d}^{s}}\right]$.

It is almost certain that the map $\Phi$ described above is the same map as the map induced by the natural inclusion of the functors $\Gamma_{(\underline{x})} \hookrightarrow \Gamma_{(y)}$. However, we do not know an easy demonstration of this and were unable to find a reference for it.

Proposition 2.8. Let $R$ be a Noetherian local ring, $d=\operatorname{dim} R$ and let $\underline{x}=$ $x_{1}, \ldots, x_{d}$ and $\underline{y}=y_{1}, \ldots, y_{d}$ be sequences of elements such that $(\underline{y}) \stackrel{A}{\subset}(\underline{x})$. Then $\Phi$ is a homomorphism from $H_{(\underline{x})}^{d}(R) \longrightarrow H_{(\underline{y})}^{d}(R)$, and $\Phi\left(\left[\frac{1}{x_{1} \cdots x_{d}}\right]\right)=\left[\frac{\operatorname{det} A}{y_{1} \cdots y_{d}}\right]$.

Proof. We adopt the notation of the above discussion. Suppose that $\left[\frac{r_{1}}{x_{1}^{n} \cdots x_{d}^{n}}\right]=$ $\left[\frac{r_{2}}{x_{1}^{n} \cdots x_{d}^{n}}\right]$, where $r_{1}, r_{2} \in R$. Then there exists a positive integer $t$ such that $\left(r_{1}-r_{2}\right)\left(x_{1} \cdots x_{d}\right)^{t} \in(\underline{x})^{[t+n]}$. We may assume that $t>n$. There exists a matrix $C$ and a positive integer $l$ such that $(\underline{y})^{[l]}=C(\underline{x})^{[t+n]}$. We have the following inclusions:

$$
(\underline{y})^{[l]} \stackrel{C}{\subset}(\underline{x})^{[t+n]} \stackrel{D_{(\underline{x})}}{\subset}(\underline{x})^{[n]} \text { and }(\underline{y})^{[l] \stackrel{D_{(\underline{y})}}{\subset}}(\underline{y})^{[s]} \stackrel{B}{\subset}(\underline{x})^{[n]},
$$

where $D_{(\underline{x})}$ is the diagonal matrix with entries $x_{i}^{t}$ and $D_{(y)}$ is the diagonal matrix with entries $y_{i}^{l-s}$.

Therefore by Corollary $2.5\left(y_{1} \cdots y_{d}\right)^{(d-1) l}\left(\operatorname{det} B\left(y_{1} \cdots y_{d}\right)^{l-s}-\left(x_{1} \cdots x_{d}\right)^{t} \operatorname{det} C\right)$ $\in(\underline{y})^{[d l]}$. Multiplying by $r_{1}-r_{2}$ we obtain

$$
\left(r_{1}-r_{2}\right) \operatorname{det} B\left(y_{1} \cdots y_{d}\right)^{d l-s}-\left(r_{1}-r_{2}\right)\left(y_{1} \cdots y_{d}\right)^{(d-1) l}\left(x_{1} \cdots x_{d}\right)^{t} \operatorname{det} C \in(\underline{y})^{[d l]} .
$$

Notice that since $\left(r_{1}-r_{2}\right)\left(x_{1} \cdots x_{d}\right)^{t} \in(\underline{x})^{[t+n]}$,

$$
\left(r_{1}-r_{2}\right)\left(y_{1} \cdots y_{d}\right)^{(d-1) l}\left(x_{1} \cdots x_{d}\right)^{t} \operatorname{det} C \in\left(y_{1} \cdots y_{d}\right)^{(d-1) l}(\underline{x})^{[t+n]} \operatorname{det} C .
$$

Also $(\underline{x})^{[t+n]} \operatorname{det} C \subset(\underline{y})^{[l]}$. Therefore

$$
\left(r_{1}-r_{2}\right)\left(y_{1} \cdots y_{d}\right)^{(d-1) l}\left(x_{1} \cdots x_{d}\right)^{t} \operatorname{det} C \in(\underline{y})^{[d l]} .
$$

Hence $\left(r_{1}-r_{2}\right) \operatorname{det} B\left(y_{1} \cdots y_{d}\right)^{d l-s} \in(\underline{y})^{[d l]}$ and thus $\left[\frac{r_{1} \operatorname{det} B}{y_{1}^{s} \cdots y_{d}^{s}}\right]=\left[\frac{r_{2} \operatorname{det} B}{y_{1}^{s} \cdots y_{d}^{s}}\right]$. So $\Phi$ is a well-defined map.

We are now ready to examine the relation between the statements in Remark 2.3. We will first assume that $y$ forms a system of parameters and determine under which conditions the maps are injective. The following proposition is proved in [12, 5.1.17]. We include a short proof for the convenience of the reader.

Proposition 2.9. Let $R$ be a Noetherian local ring, $d=\operatorname{dim} R$ and let $\underline{x}=$ $x_{1}, \ldots, x_{d}$ be a system of parameters. Let $\underline{y}=y_{1}, \ldots, y_{d}$ be a sequence of elements such that $(y) \stackrel{A}{\complement}(\underline{x})$. If $y$ forms a system of parameters, then the map

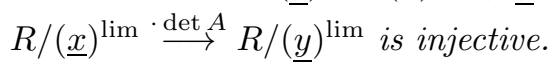


Proof. Let $r \in R$ be such that $r \cdot \operatorname{det} A \in(y)^{\lim }$. Then there exists a positive integer $s$ such that $r \operatorname{det} A\left(y_{1} \cdots y_{d}\right)^{s-1} \in(\underline{y})^{[s]}$. Since $\underline{y}$ is a system of parameters, there exist a matrix $B$ and a positive integer $t$ such that $(\underline{x})^{[t]}=B(\underline{y})^{[s]}$. Hence we have the following inclusions:

$$
(\underline{x})^{[t]} \stackrel{B}{\subset}(\underline{y})^{[s]} \stackrel{C}{\subset}(\underline{y}) \stackrel{A}{\subset}(\underline{x}) \text { and }(\underline{x})^{[t]} \stackrel{D}{\subset}(\underline{x}),
$$

where $C$ is the diagonal matrix with entries $y_{i}^{s-1}$ and $D$ is the diagonal matrix with entries $x_{i}^{t-1}$. Let $E=A C B$. By Corollary 2.5 we obtain

$$
\left(x_{1} \cdots x_{d}\right)^{t(d-1)}(\operatorname{det} E-\operatorname{det} D) \in(\underline{x})^{[t d]} .
$$

Hence

$$
\left(x_{1} \cdots x_{d}\right)^{t(d-1)} \operatorname{det} B\left(y_{1} \cdots y_{d}\right)^{s-1} \operatorname{det} A-\left(x_{1} \cdots x_{d}\right)^{t(d-1)}\left(x_{1} \cdots x_{d}\right)^{t-1} \in(\underline{x})^{[t d]}
$$

and thus $r\left(x_{1} \cdots x_{d}\right)^{t(d-1)} \operatorname{det} B\left(y_{1} \cdots y_{d}\right)^{s-1} \operatorname{det} A-r\left(x_{1} \cdots x_{d}\right)^{t d-1} \in(\underline{x})^{[t d]}$. Again,

$$
r\left(x_{1} \cdots x_{d}\right)^{t(d-1)} \operatorname{det} B\left(y_{1} \cdots y_{d}\right)^{s-1} \operatorname{det} A \in\left(x_{1} \cdots x_{d}\right)^{t(d-1)} \operatorname{det} B(\underline{y})^{[s]} .
$$

On the other hand $\left(x_{1} \cdots x_{d}\right)^{t(d-1)} \operatorname{det} B(y)^{[s]} \subset\left(x_{1} \cdots x_{d}\right)^{t(d-1)}(\underline{x})^{[t]} \subset(\underline{x})^{[t d]}$. Therefore $r\left(x_{1} \cdots x_{d}\right)^{t d-1} \in(\underline{x})^{[t d]}$, and thus $r \in(\underline{x})^{\text {lim }}$. Hence the map is injective.

Remark 2.10. Let $R$ be a Noetherian local ring, $d=\operatorname{dim} R$ and let $\underline{x}=x_{1}, \ldots, x_{d}$ and $\underline{y}=y_{1}, \ldots, y_{d}$ be sequences of elements such that $(\underline{y}) \stackrel{A}{\subset}(\underline{x})$. Then for any positive integer $n$ there exists a positive integer $s$ such that $(y)^{[s]} \subset(\underline{x})^{[n]}$. Then the map $H_{(\underline{x})}^{d}(R) \longrightarrow H_{(\underline{y})}^{d}(R)$ is the same as the map $H_{(\underline{x})^{[n]}}^{d}(R) \longrightarrow H_{(\underline{y})^{[s]}}^{d}(R)$.

Similarly, we obtain:

Proposition 2.11. Let $R$ be a Noetherian local ring, $d=\operatorname{dim} R$ and let $\underline{x}=$ $x_{1}, \ldots, x_{d}$ be a system of parameters. Let $\underline{y}=y_{1}, \ldots, y_{d}$ be a sequence of elements such that $(\underline{y}) \stackrel{A}{\subset}(\underline{x})$. If $\underline{y}$ forms a system of parameters, then the map $H_{(\underline{x})}^{d}(R) \longrightarrow$ $H_{(\underline{y})}^{d}(R)$ defined as in Discussion 2.7 is injective.

Proof. This follows immediately from Proposition 2.9. suppose that $\Phi\left(\left[\frac{r}{x_{1}^{n} \cdots x_{d}^{n}}\right]\right)$ $=0$. Set $u=\left[\frac{1}{x_{1}^{n} \cdots x_{d}^{n}}\right]$. We recall how $\Phi$ is defined: since $(\underline{y}) \subset(\underline{x})$ there

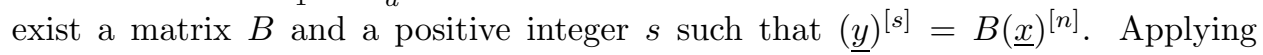
Proposition 2.9 to the two systems of parameters $(\underline{y})^{[s]} \stackrel{\bar{B}}{\subset}(\underline{x})^{[n]}$ yields that $r u=0$.

To see this notice that $H_{(\underline{x})}^{d}(R)=H_{(\underline{x})^{[n]}}^{d}(R)=\bigcup_{n \in \mathbb{N}} R /\left((\underline{x})^{[n]}\right)^{\lim }$. Let $s(n)=$ $s$ be the integer that corresponds to $n$ as above. Applying Proposition 2.9 and Remark 2.10 to the two systems of parameters $(\underline{y})^{[s(n)]} \stackrel{B}{\subset}(\underline{x})^{[n]}$, we have

$$
\begin{aligned}
H_{(\underline{x})}^{d}(R)=H_{(\underline{x})^{[n]}}^{d}(R) & =\bigcup_{n \in \mathbb{N}} R /\left((\underline{x})^{[n]}\right)^{\lim } \hookrightarrow \bigcup_{s(n) \in \mathbb{N}} R /\left((\underline{y})^{[s(n)]}\right)^{\lim } \\
& \hookrightarrow H_{(\underline{y})^{[s]}}^{d}(R)=H_{(\underline{y})}^{d}(R) .
\end{aligned}
$$




\section{Positive Characteristic}

In this section we are concerned with the maps (3) and (4) in Remark 2.3. Recall that $*$ denotes the tight closure.

Proposition 3.1. Let $R$ be a Noetherian local ring of positive characteristic p, $d=\operatorname{dim} R$ and let $\underline{x}=x_{1}, \ldots, x_{d}$ and $\underline{y}=y_{1}, \ldots, y_{d}$ be sequences of elements such that $(\underline{y}) \stackrel{A}{\subset}(\underline{x})$. Then the map $R /(\underline{x})^{*} \stackrel{\cdot \operatorname{det} A}{\longrightarrow} R /(\underline{y})^{*}$ is well-defined.

Proof. Let $z \in(\underline{x})^{*}$. Then there exists an element $c \in R^{0}$ such that $c z^{q} \in(\underline{x})^{[q]}$ for every $q=p^{e}$ large. Since $(\underline{y}) \stackrel{A}{\subset}(\underline{x})$ then $(\underline{y})^{[q]}{\stackrel{A}{A^{[q]}}}^{\subset}(\underline{x})^{[q]}$, where $A^{[q]}=\left(a_{i j}^{q}\right)$. Notice that $(\operatorname{det} A)^{q}=\operatorname{det}\left(A^{[q]}\right)$. Therefore $c(\operatorname{det} A)^{q} z^{q} \in(y)^{[q]}$ and thus $(\operatorname{det} A) z \in$ $(\underline{y})^{*}$.

The following lemma is well-known in the Noetherian case, by linkage theory. We need it in the context of a non-Noetherian ring, however. The proof we give is not the standard one.

Lemma 3.2. Let $R$ be a commutative ring with identity and let $I$ be an ideal. Suppose that $\underline{y}=y_{1}, \ldots, y_{d}$ is a regular sequence and $(y) \subset I$. Let $0 \longrightarrow R^{n} \longrightarrow$ $\cdots \longrightarrow R \longrightarrow R / I \longrightarrow 0$ be a free resolution of $R / \bar{I}$. There is a comparison map between the Koszul complex on $y_{1}, \ldots, y_{d}$ to this free resolution induced by the natural surjective map of $R / \underline{y}$ to $R / I$. Let the last map in the comparison be given by a column vector as below:

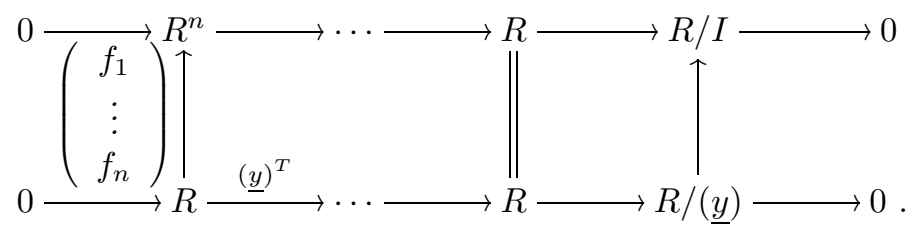

Then $(\underline{y}): I=(\underline{y})+(\underline{f})$, where $\underline{f}=f_{1}, \ldots, f_{n}$.

Proof. First notice that $R$ need not be Noetherian. To see that $(\underline{y})+(\underline{f}) \subset(\underline{y}): I$, let $i \in I$ and extend the natural map $R / I \stackrel{\cdot i}{\longrightarrow} R / I$ given by multiplication by $i$ to a map from the free resolution of $R / I$ to the free resolution of $R / I$ as in the diagram below. The composition of the map $R /(\underline{y}) \longrightarrow R / I$ with the map $R / I \stackrel{\cdot i}{\longrightarrow} R / I$ is the zero map. We begin defining a homotopy by taking $\delta_{0}=0$ :

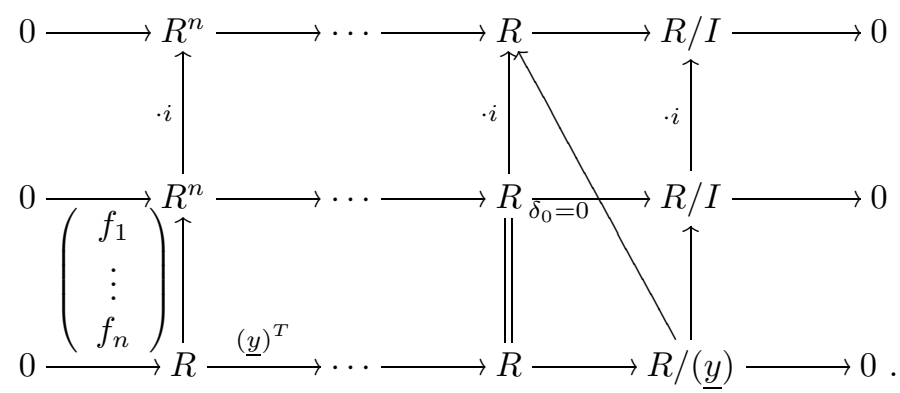

By Lemma 2.4 we may extend the homotopy to obtain $i\left(f_{1}, \ldots, f_{d}\right) \subset\left(y_{1}, \ldots, y_{d}\right)$. 
For the other inclusion let $s \in(y): I$ and consider the map $R / I \stackrel{\cdot s}{\longrightarrow} R /(y)$ given by multiplication by $s$. Extending this map we obtain the following diagram:

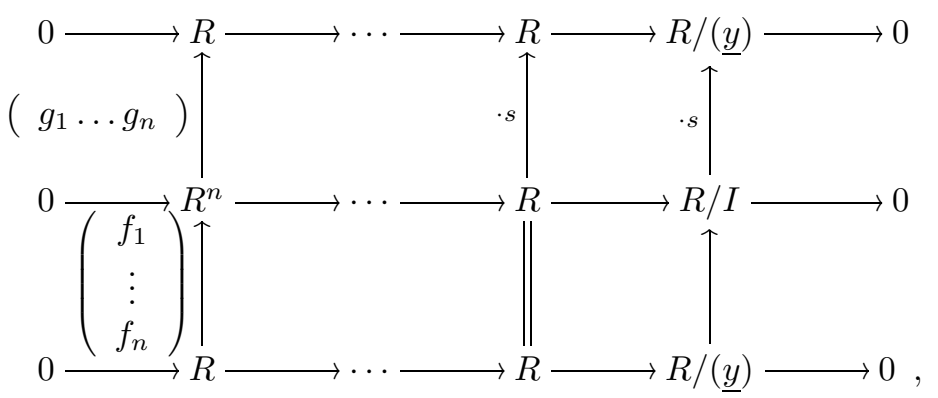

for some elements $g_{1}, \ldots, g_{n} \in R$.

On the other hand we consider the map $R /(y) \stackrel{\cdot s}{\longrightarrow} R /(y)$ given by multiplication by $s$. Extending this map we obtain the following diagram:

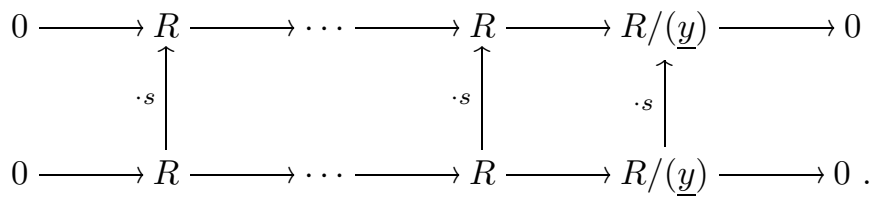

Combining the two diagrams we can define a homotopy by taking $\delta_{0}=0$, and by Lemma 2.4 we can extend the homotopy:

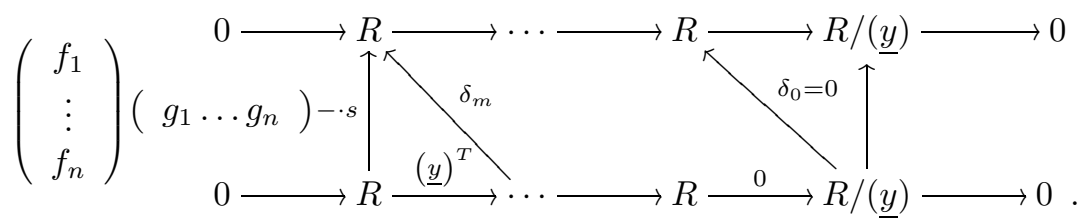

Therefore $s-\sum_{i=1}^{n} f_{i} g_{i} \in\left(y_{1}, \ldots, y_{d}\right)$ and $s \in(\underline{y})+(\underline{f})$.

Proposition 3.3. Let $R$ be an excellent local ring of positive characteristic $p$, $d=\operatorname{dim} R$, and $\underline{x}=x_{1}, \ldots, x_{d}$ be a system of parameters. Let $\underline{y}=y_{1}, \ldots, y_{d}$ be a sequence of elements such that $(\underline{y}) \stackrel{A}{\subset}(\underline{x})$. If $\underline{y}$ forms a system of parameters, then the map $R /(\underline{x})^{*} \stackrel{\operatorname{det} A}{\longrightarrow} R /(\underline{y})^{*}$ is injective.

Proof. We can first pass to the completion and thus assume that $R$ is complete. By Proposition 3.1 the map $R /(\underline{x})^{*} \stackrel{\operatorname{det} A}{\longrightarrow} R /(y)^{*}$ is well-defined and therefore $(\underline{x})^{*} \subset(\underline{y})^{*}: \operatorname{det} A$. Let $r \in(y)^{*}: \operatorname{det} A$. To show that $r \in(\underline{x})^{*}$ it is enough to show that the image of $r$ in $R / P$ is in the tight closure of $((\underline{x})+P) / P$ for every minimal prime $P$ of $R$ by [6. Proposition 4.1]. Thus we may assume that $R$ is a domain.

Since $r \operatorname{det} A \in(\underline{y})^{*}$ and $(\underline{y})^{*} \subset(\underline{y}) R^{+}$, it follows that $r \operatorname{det} A \in(\underline{y}) R^{+}$. In $R^{+}$ both $\underline{x}$ and $y$ form a regular sequence. Therefore $(y) R^{+}: \operatorname{det} A=(\underline{x}) R^{+}$since $R^{+}$ is Cohen-Macaulay or by Lemma 3.2, Hence $r \in(\underline{x}) R^{+} \cap R=(\underline{x})^{*}$, where the equality follows by [11, Theorem 5.1]. 
The fact that parameters form a regular sequence in $R^{+}$immediately gives injectivity on local cohomology:

Proposition 3.4. Let $R$ be a Noetherian local domain of positive characteristic $p$, $d=\operatorname{dim} R$, and $\underline{x}=x_{1}, \ldots, x_{d}$ be a system of parameters. Let $\underline{y}=y_{1}, \ldots, y_{d}$ be a sequence of elements such that $(\underline{y}) \stackrel{A}{\subset}(\underline{x})$. If $\underline{y}$ forms a system of parameters, then the $\operatorname{map} H_{(\underline{x})}^{d}\left(R^{+}\right) \longrightarrow H_{(\underline{y})}^{d}\left(R^{+}\right)$is injective.

Proof. This follows as in Proposition [2.11] suppose that $\Phi\left(\left[\frac{r}{x_{1}^{n} \cdots x_{d}^{n}}\right]\right)=0$. Set $u=\left[\frac{1}{x_{1}^{n} \cdots x_{d}^{n}}\right]$. We recall how $\Phi$ is defined: since $(\underline{y}) \subset(\underline{x})$ there exist a matrix $B$ and a positive integer $s$ such that $(\underline{y})^{[s]}=B(\underline{x})^{[n]}$. Since parameters form a regular sequence in $R^{+},(\underline{y})^{[s]}: \operatorname{det} B=(\underline{x})^{[n]}$, and then it follows that $r u=0$.

\section{The OnE-Dimensional CASE}

In this section we prove two main results: that the injectivity of either map $R /(x) \stackrel{\cdot u}{\longrightarrow} R /(y)$ or $R /(x)^{\lim } \stackrel{\cdot u}{\longrightarrow} R /(y)^{\lim }$ forces $y$ to be a parameter. It is interesting that even this simple case is not obvious.

Theorem 4.1. Let $R$ be a one-dimensional Noetherian local ring. Let $x$ be a parameter, and let $y=u x$. If the map $R /(x) \stackrel{\cdot u}{\longrightarrow} R /(y)$ is injective, then $y$ is a parameter.

Proof. Suppose that $x$ is a parameter and that the map $R /(x) \stackrel{\cdot u}{\longrightarrow} R /(y)$ is injective. Since $x$ is a parameter, $0: x$ has finite length. Consider the following exact sequence:

$$
0 \longrightarrow(0:(x, u)) \longrightarrow 0: x \stackrel{\cdot u}{\longrightarrow} 0: x \longrightarrow \frac{0: x}{u(0: x)} \longrightarrow 0
$$

Computing length we obtain that $\lambda\left(\frac{0: x}{u(0: x)}\right)=\lambda((0:(x, u)))$. Suppose that $u$ is not a parameter. Then there exists a minimal prime $P$ such that $u \in P$. Hence $0:(0: u) \subset P$ and in particular $\operatorname{dim}((0: u))>0$, and consequently $e(x ;(0: u))>0$. On the other hand, $e(x ;(0: u))=\lambda\left(\frac{0: u}{x(0: u)}\right)-\lambda\left(0:{ }_{(0: u)} x\right)>0$. Since $0:(0: u) x=$ $0:(x, u)$, by the above computations we obtain $\lambda\left(\frac{0: u}{x(0: u)}\right)>\lambda\left(\frac{0: x}{u(0: x)}\right)$.

Now since the map is injective we have $0: u \subset(x)$ and thus $0: u=x(0: x u)$. Hence

$$
\frac{0: u}{x(0: u)}=\frac{x(0: x u)}{x(0: u)} \simeq \frac{0: x u}{(0: u)+(0: x)} \simeq \frac{u(0: x u)}{u(0: x)} \subset \frac{0: x}{u(0: x)}
$$

Therefore $\lambda\left(\frac{0: x}{u(0: x)}\right) \geq \lambda\left(\frac{u(0: x u)}{u(0: x)}\right)=\lambda\left(\frac{0: u}{x(0: u)}\right)$, which is a contradiction. Thus $u$ must be a parameter, and in conclusion $y$ is also a parameter.

Corollary 4.2. Let $R$ be a one-dimensional Noetherian local ring. Let $x$ be a parameter and let $y=u x$. The $\operatorname{map} R /(x) \stackrel{\cdot u}{\longrightarrow} R /(y)$ is injective if and only if the map $R /(u) \stackrel{\cdot x}{\longrightarrow} R /(y)$ is injective. 
Proof. Notice that it suffices to show one direction. Suppose that $R /(x) \stackrel{\cdot u}{\longrightarrow} R /(y)$ is injective. Then by Theorem $4.1 u$ is also a parameter. By the proof of Theorem 4.1 we see that by symmetry one has

$$
\frac{0: u}{x(0: u)} \simeq \frac{x(0: x u)}{x(0: u)} \simeq \frac{0: x u}{(0: x)+(0: u)} \simeq \frac{u(0: x u)}{u(0: x)} \subset \frac{0: x}{u(0: x)} .
$$

But since $\lambda\left(\frac{0: u}{x(0: u)}\right)=\lambda\left(\frac{0: x}{u(0: x)}\right), \frac{u(0: x u)}{u(0: x)} \simeq \frac{0: x}{u(0: x)}$. Therefore $0: x \subset(u)$, i.e. $R /(u) \stackrel{\cdot x}{\longrightarrow} R /(y)$ is injective.

The converse of Theorem 4.1 does not hold in general. The following example was shown to us by R. Heitmann.

Example 4.3. Let $R=k[x, u] /\left(((x+u) u)^{3}, x(x+u)^{2} u^{2}\right)$, where $k$ is a field and char $k=2$. Notice that $R$ is a one-dimensional ring, where $x$ is a parameter. Let $y=x^{2}$ also be a parameter. Then one can see that $(y):(x)=\left(x, u^{4}\right)$, which means that the map $R /(x) \stackrel{\cdot x}{\longrightarrow} R /(y)$ is not injective.

In fact, in Section 6 we will prove in arbitrary dimension that if the maps are injective for all parameters, then the ring must be Cohen-Macaulay.

Theorem 4.4. Let $R$ be a one-dimensional Noetherian local ring. Let $x$ be a parameter and let $y=u x$. Then $y$ is a parameter if and only if the map $R /(x)^{\lim } \stackrel{\cdot u}{\longrightarrow}$ $R /(y)^{\lim }$ is injective.

Proof. First notice that the forward direction follows from Proposition 2.9. So

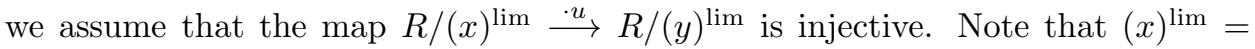
$\left(x^{n+1}\right): x^{n}$ for all large $n$, which in turn is equal to $(x)+\left(0: x^{n}\right)$ for large $n$. Similarly, $(y)^{\lim }=(y)+\left(0: y^{n}\right)$ for large enough $n$. We can analyze these ideals using a primary decomposition of $(0)$. Let $0=q_{1} \cap q_{2} \cap \ldots \cap q_{s} \cap J$, where $\sqrt{q_{i}}=p_{i}$ are distinct minimal primes and $\sqrt{J}=\mathfrak{m}$. We wish to prove that $y$ is a parameter. Assume not. Then $y$ is in at least one minimal prime. Let $y \in p_{1} \cap \ldots \cap p_{t}$ and $y \notin p_{t+1} \cup \ldots \cup p_{s}$. We see that $(x)+\left(0: x^{n}\right)=(x)+q_{1} \cap q_{2} \cap \ldots \cap q_{s}$ and $(y)+\left(0: y^{n}\right)=(y)+q_{t+1} \cap \ldots \cap q_{s}$. The assumption on the injectivity of the map then becomes that $\left((y)+q_{t+1} \cap \ldots \cap q_{s}\right): u \subset(x)+q_{1} \cap q_{2} \cap \ldots \cap q_{s}$. Since $u$ is not in $p_{t+1} \cup \ldots \cup p_{s}$, it follows that $\left((y)+q_{t+1} \cap \ldots \cap q_{s}\right): u=(x)+q_{t+1} \cap \ldots \cap q_{s} \subset$ $(x)+q_{1} \cap q_{2} \cap \ldots \cap q_{s}$. In particular, $q_{t+1} \cap \ldots \cap q_{s} \subset x\left(q_{t+1} \cap \ldots \cap q_{s}\right)+q_{1} \cap q_{2} \cap \ldots \cap q_{s}$, which by Nakayama's lemma shows that $t=0$. It follows that $y$ is a parameter.

Discussion 4.5. It is natural to believe that the one-dimensional case above would at least prove the higher-dimensional case when the matrix $A$ is a diagonal matrix, i.e., the case in which $x_{1}, \ldots, x_{d}$ are parameters and $y_{i}=u_{i} x_{i}$. In this case our assumption would be that the map $R /(\underline{x}) \stackrel{u_{1} \cdots u_{d}}{\rightarrow} R /(\underline{y})$ is injective. One is tempted to break this up into several maps by changing one $x_{i}$ at a time. Although this works for one step, it seems to break down even in dimension 2 and gives a good idea of the difficulty in extending to higher dimensions.

\section{Higher Dimensions}

We would like to extend Theorem 4.4 to higher dimensions. We are able to do so in the case where the ring is analytically irreducible, as well as in the case in which the system of parameters $\underline{x}$ lies deep inside the maximal ideal. 
Proposition 5.1. Let $R$ be an analytically irreducible local ring of equicharacteristic and $d=\operatorname{dim} R$, or of mixed characteristic and of dimension $d$ at most 3 . Let $\underline{x}=x_{1}, \ldots, x_{d}$ be a system of parameters. Let $\underline{y}=y_{1}, \ldots, y_{d}$ be a sequence of elements such that $(\underline{y}) \stackrel{A}{\subset}(\underline{x})$. Then $\underline{y}$ forms a system of parameters if and only if the

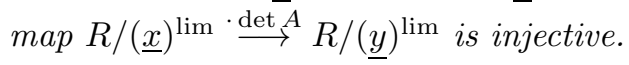

Proof. We may assume that $R$ is a complete domain. The forward direction is already covered in Proposition 2.9 .

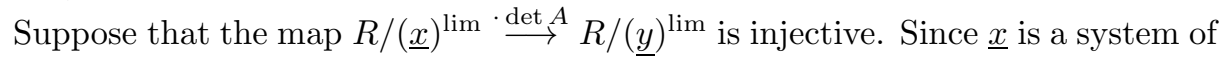
parameters, then $(\underline{x})^{\lim } \neq R$. This is the monomial conjecture of Hochster, which is true in either equicharacteristic [5] or in dimension at most three [4]. As the map $R /(\underline{x})^{\lim } \stackrel{\operatorname{det} A}{\longrightarrow} R /(\underline{y})^{\lim }$ is injective, then $R /(\underline{y})^{\lim } \neq 0$. Also, notice that $R /(\underline{y})^{\lim }$ injects naturally into $H_{(\underline{y})}^{d}(R)$, and thus $H_{(\underline{y})}^{d}(R) \neq 0$. Therefore by the Hartshorne-Lichtenbaum Vanishing Theorem there exists a minimal prime $P$ of $R$ with $\operatorname{dim} R / P=\operatorname{dim} R$ such that $\operatorname{dim}(R /((\underline{y})+P))=0$. Since $R$ is a domain it follows that $\operatorname{dim} R /(\underline{y})=0$, and thus $\underline{y}$ is a system of parameters.

If $R$ is not analytically irreducible, then unfortunately we cannot reach the same conclusion. See Example 6.1 in the last section. However, we can say the following:

Theorem 5.2. Let $(R, \mathfrak{m})$ be a Noetherian local ring, $d=\operatorname{dim} R$, and let $\underline{x}=$ $x_{1}, \ldots, x_{d}$ be a system of parameters. Let $\underline{y}=y_{1}, \ldots, y_{d}$ be a sequence of elements such that $(\underline{y}) \stackrel{A}{\subset}(\underline{x})$. There exists an integer $\ell$ such that if $(\underline{x}) \subset \mathfrak{m}^{\ell}$, then the map $R /(\underline{x})^{\lim } \stackrel{\operatorname{det} A}{\longrightarrow} R /(\underline{y})^{\lim }$ is injective if and only if the map $H_{(\underline{x})}^{d}(R) \longrightarrow H_{(\underline{y})}^{d}(R)$ as in Discussion 2.7 is injective.

Proof. By [3, Lemma 3.12] there exists an integer $\ell$ such that if $\underline{x}$ is system of parameters in $\hat{R}$ such that $(\underline{x}) \subset \mathfrak{m}^{\ell} \hat{R}$, then the map $\operatorname{Soc}(\hat{R} /(\underline{x})) \longrightarrow \operatorname{Soc}\left(H_{(\underline{x})}^{d}(\hat{R})\right)$ is surjective. Notice that $\underline{x}$ is a system of parameters in $\hat{R}$ if and only if $\underline{x}$ is a system of parameters in $R$. Also the map $H_{(\underline{x})}^{d}(R) \longrightarrow H_{(\underline{y})}^{d}(R)$ is injective if and only if $H_{(\underline{x})}^{d}(\hat{R}) \longrightarrow H_{(\underline{y})}^{d}(\hat{R})$ is injective. Therefore without loss of generality we may assume that $R$ is complete.

Suppose that $(\underline{x}) \subset \mathfrak{m}^{\ell}$. Notice that $R /(\underline{x})^{\lim }$ injects naturally into $H_{(\underline{x})}^{d}(R)$ and similarly $R /(\underline{y})^{\lim }$ injects naturally into $H_{(\underline{y})}^{d}(R)$. By Proposition 2.8 there exists a homomorphism $\Phi: H_{(\underline{x})}^{d}(R) \longrightarrow H_{(\underline{y})}^{d}(R)$, and moreover the following diagrams commute:

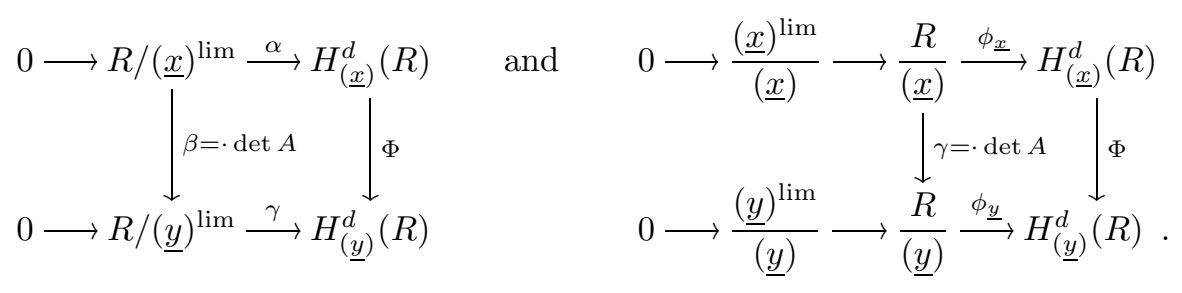

Notice that if $\Phi$ is injective, then clearly $\beta$ is injective. Suppose that the map $R /(\underline{x})^{\lim \cdot \operatorname{det} A} A R /(\underline{y})^{\lim }$ is injective. Suppose that $\Phi$ is not injective. Since $H_{(\underline{x})}^{d}(R)$ 
is an Artinian module, $H_{(\underline{x})}^{d}(R)$ is essential over $\operatorname{Soc}\left(H_{(\underline{x})}^{d}(R)\right)$. Hence there exists a nonzero element $f \in \operatorname{ker}(\Phi) \cap \operatorname{Soc}\left(H_{(\underline{x})}^{d}(R)\right)$. By [프, Lemma 3.12] the map $\operatorname{Soc}(R /(\underline{x})) \stackrel{\phi_{\underline{x}}}{\rightarrow} \operatorname{Soc}\left(H_{(\underline{x})}^{d}(R)\right)$ is surjective. Hence there exists $g \in \operatorname{Soc}(R /(\underline{x}))$ such that $\phi_{\underline{x}}(g)=f$. But as the second diagram commutes, $\phi_{\underline{y}}(\gamma(g))=\Phi\left(\phi_{\underline{x}}(f)\right)$, or in other words $\phi_{\underline{y}}((\operatorname{det} A) g)=0$. Since $\operatorname{ker} \phi_{\underline{y}}=\frac{(\underline{y})^{\lim }}{(\underline{y})}$, it follows that $(\operatorname{det} A) g \in$ $(\underline{y})^{\lim }$. Thus since $\beta$ is injective, $g \in(\underline{x})^{\lim }$, or in other words, $g \in \operatorname{ker} \phi_{\underline{x}}$ and hence $\bar{f}=0$, which is a contradiction. Therefore, $\Phi$ is also injective.

Theorem 5.3. Let $(R, \mathfrak{m})$ be an equidimensional Noetherian local ring and let $d=\operatorname{dim} R$. There exists an integer $\ell$ with the following property: whenever $\underline{x}=$ $x_{1}, \ldots, x_{d}$ is a system of parameters with $(\underline{x}) \subset \mathfrak{m}^{\ell}$ and $\underline{y}=y_{1}, \ldots, y_{d}$ is a sequence of elements such that $(\underline{y}) \stackrel{A}{\subset}(\underline{x})$, then $\underline{y}$ forms a system of parameters if and only if the map $H_{(\underline{x})}^{d}(R) \longrightarrow \bar{H}_{(\underline{y})}^{d}(R)$ as in Discussion 2.7 is injective.

Proof. As in the proof of Theorem 5.2 we may pass to the completion $\hat{R}$ and assume that $R$ is complete. Let $\ell$ be the integer as in [3, Lemma 3.12] such that $(\underline{x}) \subset \mathfrak{m}^{\ell}$ and the $\operatorname{map} \operatorname{Soc}(R /(\underline{x})) \longrightarrow \operatorname{Soc}\left(H_{(\underline{x})}^{d}(R)\right)$ is surjective.

First we assume that $\underline{y}$ is a system of parameters. Then by Proposition 2.9

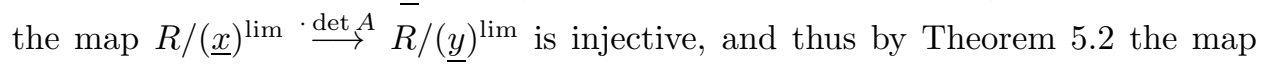
$H_{(\underline{x})}^{d}(R) \longrightarrow H_{(\underline{y})}^{d}(R)$ as in Discussion 2.7 is injective.

Suppose the map $H_{(\underline{x})}^{d}(R) \longrightarrow H_{(\underline{y})}^{d}(R)$ as in Discussion 2.7 is injective. Since $R$ is equidimensional, to show that $\underline{y}$ is a system of parameters it suffices to show that $\underline{y}$ is a system of parameters in $\bar{R}=R / P$ for every minimal prime $P \square$ Suppose that this is not true. Then there exists a minimal prime $P$ such that $H_{(\underline{y})}^{d}(\bar{R})=0$, where $\bar{R}=R / P$. Let $P=0: c$ for some $c \in R$. We have the short exact sequence

$$
0 \longrightarrow R /(0: c) \longrightarrow R \stackrel{\pi}{\longrightarrow} R /(c) \longrightarrow 0 .
$$

Since $H_{(\underline{y})}^{d}(\bar{R})=0$ we obtain the sequence

$$
H_{(\underline{x})}^{d}(R) \stackrel{\Phi}{\hookrightarrow} H_{(\underline{y})}^{d}(R) \stackrel{\pi}{\hookrightarrow} H_{(\underline{y})}^{d}(R /(c)) \longrightarrow 0 .
$$

We claim that $c H_{(\underline{x})}^{d}(R)=0$. Let $f \in H_{(\underline{x})}^{d}(R)$. Then $\pi(\Phi(c f))=\pi(c \Phi(f))=$ $c \pi(\Phi(f))$. But $c H_{(\underline{y})}^{d}(R / c)=0$, so $c \pi(\Phi(f))=0$. On the other hand, though, both $\pi$ and $\Phi$ are injective, and hence $c f=0$, which shows the claim.

We note now that since $c H_{(\underline{x})}^{d}(R)=0$, it follows that $c\left(H_{(\underline{x})}^{d}(R)\right)^{\vee}=0$. But the dual of the top local cohomology is $\omega_{R}$, the canonical module of $R$. Thus $c \in \operatorname{ann} \omega_{R}$, which is a contradiction, since by [7, Remark 2.2] $\operatorname{ann} \omega_{R}=\{r \in R \mid \operatorname{dim} R /(0: r)$ $<d\}$.

Corollary 5.4. Let $(R, \mathfrak{m})$ be an equidimensional Noetherian local ring and let $d=\operatorname{dim} R$. There exists an integer $\ell$ with the following property: whenever $\underline{x}=$ $x_{1}, \ldots, x_{d}$ is a system of parameters with $(\underline{x}) \subset \mathfrak{m}^{\ell}$ and $\underline{y}=y_{1}, \ldots, y_{d}$ is a sequence

\footnotetext{
${ }^{1}$ We wish to thank N. T. Cuong for pointing out to us that the ring needs to be equidimensional in this theorem. Note that Cuong and Nhan have also studied the limit closure of parameter ideals in 1] under a different name.
} 
of elements such that $(y) \stackrel{A}{\subset}(\underline{x})$, then $y$ forms a system of parameters if and only if

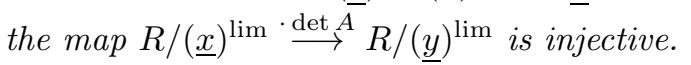

Proof. Simply combine the last two theorems.

\section{Examples, EXtensions, And QUestions}

In this section we conclude with several examples which show that the hypotheses of several of the theorems are necessary, and we consider some extensions of our results and list some open questions.

The first example shows that the condition that the parameters are in a deep enough power of the maximal ideal is necessary in Corollary [5.4.

Example 6.1. Let $R=\frac{k[a, b, c, d]}{(a, b) \cap(c, d)}$, where $k$ is a field. Notice that $R$ is an equidimensional ring. Let $x_{1}=a+c, x_{2}=b+d$ and $y_{1}=a^{2}, y_{2}=b^{2}$. We claim that $\underline{x}=x_{1}, x_{2}$ is a system of parameters, $(y)=\left(y_{1}, y_{2}\right) \subset(\underline{x})$, the map

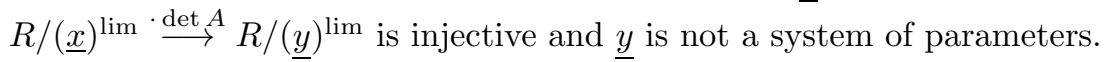

First note that it is straightforward to see that $\underline{x}=x_{1}, x_{2}$ is a system of parameters, $(\underline{y})=\left(y_{1}, y_{2}\right) \subset(\underline{x})$ and $y$ is not a system of parameters. We now claim that $(\underline{x})^{\lim }=\mathfrak{m}=(a, b, c, d)$. Notice that $\mathfrak{m}\left(x_{1} x_{2}\right) \subset\left(x_{1}^{2}, x_{2}^{2}\right)$ and thus $\mathfrak{m} \subset(\underline{x})^{\lim }$. Since $(\underline{x})^{\lim } \neq R,(\underline{x})^{\lim }=\mathfrak{m}$.

We also claim that $(\underline{y})^{\lim }=\left(a^{2}, b^{2}, c, d\right)$. Notice that $\left(a^{2}, b^{2}, c, d\right)\left(a^{2} b^{2}\right) \subset$ $\left(a^{4}, b^{4}\right)$. On the other hand suppose $z\left(a^{2 n} b^{2 n}\right) \subset\left(a^{2 n+2}, b^{2 n+2}\right)$. We consider the $\operatorname{ring} \bar{R}=R /(c, d) \simeq k[a, b]$. Let - denote the images in $\bar{R}$. Then $\bar{z} \subset\left(a^{2}, b^{2}\right) \bar{R}$. Thus $z \in\left(a^{2}, b^{2}, c, d\right)$, and therefore $\left(a^{2}, b^{2}, c, d\right)=(y)^{\lim }$.

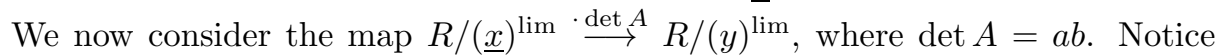
that 1 maps to $a b$ and the map is clearly injective.

The next example deals with the question of whether or not the injectivity of the map in the original situation of Dutta and Roberts actually forces the ring to be Cohen-Macaulay. The answer is no, as the first example shows, but it is yes if one requires injectivity for all systems of parameters.

Example 6.2. Let $R=k[x, z] /\left(x^{2} z, z^{2}\right)$ be a one-dimensional ring. Then $R$ is not Cohen-Macaulay. However, $x$ and $y=x^{2}$ are parameters, and $x^{2}:_{R} x=(x)$.

Proposition 6.3. Let $R$ be a Noetherian local ring and let $d=\operatorname{dim} R$. Suppose that for every system of parameters $\underline{x}=x_{1}, \ldots, x_{d}$ and every positive integer $t$, the map $R /(\underline{x}) \stackrel{\left(x_{1} \cdots x_{d}\right)}{\longrightarrow}{ }^{t-1} R /(\underline{x})^{[t]}$ is injective. Then $R$ is Cohen-Macaulay.

Proof. Notice that $(\underline{x})^{\lim }=(\underline{x})^{[t]}:\left(x_{1} \cdots x_{d}\right)^{t-1}$ for some $t \in \mathbb{N}$. Since $\underline{x}$ is a system of parameters, so is $\underline{x}^{[t]}$. Therefore by assumption the map $R /(\underline{x}) \stackrel{\operatorname{det} D}{\longrightarrow} R /(\underline{x})^{[t]}$ is injective, where now $D$ is the diagonal matrix with diagonal entries $x_{i}^{t-1}$. Then $(\underline{x})=(\underline{x})^{[t]}: \operatorname{det} D=(\underline{x})^{[t]}:\left(x_{1} \cdots x_{d}\right)^{t-1}$, since the map is injective. Hence $(\underline{x})=(\underline{x})^{\lim }$, and by [8, Proposition 2.3] (see also [12, Theorem 5.2.3]) $R$ is then Cohen-Macaulay.

We close with two additional questions which are suggested by the work in this paper. 
Question 6.4. Let $R$ be a Noetherian local ring. Suppose that $u$ is in some minimal prime $P$ such that the dimension of $R / P$ is the same as that of $R$. Can $0: u$ ever be in an ideal generated by a system of parameters?

We proved that the answer is 'no' in dimension one, and our calculations strongly suggest the answer is always 'no'. A related observation is due to Strooker and Simon [10. They prove the following: let $R$ be a Gorenstein local ring and let $A=R / I$ where $I$ is a nonzero ideal in $R$ consisting of zero divisors. Set $J=0: I$. Then $A$ satisfies the monomial conjecture if and only if the ideal $J$ is not contained in any parameter ideal of $R$.

Another question was suggested to us by Bernd Ulrich.

Question 6.5. Our main question can be rephrased to say that $(\underline{y}): \operatorname{det} A=(\underline{x})$ should imply that $y$ are parameters. This statement, in the Cohen-Macaulay case, is equivalent to the dual statement that $(\underline{y}):(\underline{x})=(\underline{y}, \operatorname{det} A)$. What is the relationship between the two in general?

Note that in the one-dimensional case, Corollary 4.2 proves the two are equivalent.

\section{REFERENCES}

[1] N. T. Cuong and L. T. Nhan, Pseudo Cohen-Macaulay and pseudo generalized CohenMacaulay modules, J. Algebra 267 (2003), no. 1, 156-177. MR.1993472 (2004f:13012)

[2] S. P. Dutta and P. C. Roberts, A characterization of systems of parameters, Proc. Amer. Math. Soc. 124 (1996), no. 3, 671-675. MR 1283547(96f:13017)

[3] S. Goto and H. Sakurai, The equality $I^{2}=Q I$ in Buchsbaum rings, Rend. Sem. Mat. Univ. Padova 110 (2003), 25-56. MR.2033000 (2004m:13061)

[4] R. Heitmann, The direct summand conjecture in dimension three, Ann. of Math. (2) 156 (2002), 695-712. MR1933722 (2003m:13008)

[5] M. Hochster, Topics in the homological theory of modules over commutative rings, CBMS 24 (1975) AMS, Providence, RI. MR0371879 (51:8096)

[6] M. Hochster and C. Huneke, Tight closure, invariant theory, and the Briançon-Skoda theorem, J. Amer. Math. Soc. 3 (1990), no. 1, 31-116. MR.1017784 (91g:13010)

[7] M. Hochster and C. Huneke, Indecomposable canonical modules and connectedness, Commutative algebra: syzygies, multiplicities, and birational algebra (South Hadley, MA, 1992), 197208, Contemp. Math., 159, Amer. Math. Soc., Providence, RI, 1994. MR.1266184(95e:13014)

[8] T. Marley, M. W. Rogers, and H. Sakurai, Gorenstein rings and irreducible parameter ideals, Proc. Amer. Math. Soc. 136 (2008), no. 1, 49-53. MR2350387 (2009b:13043)

[9] G. Scheja and U. Storch, Uwe Differentielle Eigenschaften der Lokalisierungen analytischer Algebren, Math. Ann. 197 (1972), 137-170. MR0306172 (46:5299)

[10] A.-M. Simon and J. Strooker, Gorenstein rings call the tune, Lect. Notes Pure Appl. Math., 244, 227-238, Chapman and Hall/CRC, Boca Raton, FL, 2006. MR2184800 (2006g:13030)

[11] K. E. Smith, Tight closure of parameter ideals. Invent. Math. 115 (1994), no. 1, 41-60. MR.1248078 (94k:13006)

[12] J. R. Strooker, Homological questions in local algebra, London Mathematical Society Lecture Note Series, 145, Cambridge University Press, Cambridge, 1990. MR1074178 (91m:13013)

Department of Mathematical Sciences, New Mexico State University, Las Cruces, New MeXico 88003

E-mail address: lfouli@math.nmsu.edu

URL: http://www.math.nmsu.edu/ lfouli

Department of Mathematics, University of Kansas, Lawrence, Kansas 66045

E-mail address: huneke@math.ku.edu

URL: http://www. math.ku.edu/ ${ }^{\text {huneke }}$ 